\title{
Neural basis of three dimensions of agitated behaviors in patients with Alzheimer disease
}

\author{
This article was published in the following Dove Press journal: \\ Neuropsychiatric Disease and Treatment \\ 17 February 2014 \\ Number of times this article has been viewed
}

\author{
Koichi Banno' \\ Shutaro Nakaaki \\ Junko Sato' \\ Katsuyoshi Torii' \\ Jin Narumoto ${ }^{3}$ \\ Jun Miyata ${ }^{4}$ \\ Nobutsugu Hirono 5 \\ Toshi A Furukawa ${ }^{6}$ \\ Masaru Mimura ${ }^{2}$ \\ Tatsuo Akechi' \\ 'Department of Psychiatry and \\ Cognitive-Behavioral Medicine, \\ Nagoya City University Graduate \\ School of Medical Sciences, \\ Nagoya, Japan; ${ }^{2}$ Department of \\ Neuropsychiatry, Keio University \\ School of Medicine, Tokyo, Japan; \\ ${ }^{3}$ Department of Psychiatry, Graduate \\ School of Medical Science, Kyoto \\ Prefectural University of Medicine, \\ ${ }^{4}$ Department of Neuropsychiatry, \\ Graduate School of Medicine, \\ Kyoto University, Kyoto, Japan; \\ ${ }^{5}$ Department of Psychology, Kobe \\ Gakuin University; Hyogo, Japan; \\ ${ }^{6}$ Departments of Health Promotion \\ and Human Behavior and of Clinical \\ Epidemiology, Graduate School of \\ Medicine/School of Public Health, \\ Kyoto University, Kyoto, Japan
}

Correspondence: Shutaro Nakaaki Department of Neuropsychiatry, Keio University School of Medicine, 35 Shinanomachi, Shinjuku-ku,

Tokyo 160-8582, Japan

Tel +8I 353633829

Fax +8I 353790187

Email hzi05510@nifty.com
Background: Agitated behaviors are frequently observed in patients with Alzheimer disease (AD). The neural substrate underlying the agitated behaviors in dementia is unclear. We hypothesized that different dimensions of agitated behaviors are mediated by distinct neural systems.

Methods: All the patients $(\mathrm{n}=32)$ underwent single photon emission computed tomography (SPECT). Using the Agitated Behavior in Dementia scale, we identified the relationships between regional cerebral blood flow ( $\mathrm{rCBF}$ ) patterns and the presence of each of three dimensions of agitated behavior (physically agitated behavior, verbally agitated behavior, and psychosis symptoms) in AD patients. Statistical parametric mapping (SPM) software was used to explore these neural correlations.

Results: Physically agitated behavior was significantly correlated with lower rCBF values in the right superior temporal gyrus (Brodmann 22) and the right inferior frontal gyrus (Brodmann 47). Verbally agitated behavior was significantly associated with lower $\mathrm{CCBF}$ values in the left inferior frontal gyrus (Brodmann 46, 44) and the left insula (Brodmann 13). The psychosis symptoms were significantly correlated with lower rCBF values in the right angular gyrus (Brodmann 39) and the right occipital lobe (Brodmann 19).

Conclusion: Our results support the hypothesis that three different agitated behaviors may represent distinct neural networks in AD patients.

Keywords: physically agitated behavior, verbally agitated behavior, psychosis, SPECT

\section{Introduction}

Agitated behaviors are frequent symptoms in patients with Alzheimer disease (AD). The reported prevalence of agitated behaviors in AD patients is approximately $30 \%-50 \%$, even among patients with a mild stage of AD. ${ }^{1,2}$ Although the concept of agitated behavior is rather vague, since it includes a wide range of inappropriate behavior (including verbal, vocal, or motor activities), ${ }^{3}$ agitated behavior is usually considered to result in serious impairments of daily functioning. Thus, agitated behaviors are very distressing to the caregivers of AD patients.

Cohen-Mansfield proposed that agitated behaviors can be classified into several different subtypes: verbally nonaggressive, verbally aggressive, physically nonaggressive, and physically aggressive. ${ }^{3}$ Most previous factor analyses have supported these dimensions. ${ }^{4,5}$ Understanding the different mechanisms of the subtypes of agitated behavior is clinically important for both clinical intervention and prognosis. Our earlier study using the Agitated Behavior in Dementia scale (ABID) ${ }^{6}$ showed that agitated behaviors in $\mathrm{AD}$ patients can be grouped according to three dimensions: physically 
agitated behavior, verbally agitated behavior, and psychosis symptoms. In our previous study, ${ }^{6}$ we demonstrated that the ABID has several clinical advantages over the CohenMansfield Agitation Inventory (CMAI) ${ }^{3-5}$ for the assessment of dementia in patients with agitated behaviors. For example, the ABID may be an appropriate assessment for community-dwelling subjects with mild to moderate levels of dementia. In contrast, the CMAI may be more appropriate for more severely disturbed nursing home residents. In terms of cognitive impairment, different types of agitated behavior are postulated to be associated with different patterns of cognitive functioning. ${ }^{3,7,8}$ Several studies have suggested that agitated behaviors are mediated by frontal lobe dysfunction. ${ }^{9,10}$ However, the neural basis of the different types of agitated behaviors remains unclear.

A few neuroimaging studies have addressed the neural basis of agitated behaviors in dementia patients. Two functional neuroimaging studies using single photon emission computed tomography (SPECT) demonstrated that hypoperfusion in either the left anterior temporal region ${ }^{11}$ or the right medial temporal region ${ }^{12}$ is chiefly responsible for agitated behaviors. Another study, using positron emission tomography (PET), demonstrated that perfusion in both the frontal cortex and the temporal cortex was correlated with the agitation score. ${ }^{13} \mathrm{~A}$ recent structural brain study using voxel-based morphometry (VBM) implied that agitation was associated with decreased gray matter in the left insula and bilateral anterior cingulate cortex. ${ }^{14}$ This variability in the brain regions responsible for agitated behaviors might have arisen, not only from the use of different methods to assess agitation but also, from the inclusion of $\mathrm{AD}$ patients with various types of agitated behaviors. In addition, this variability in the brain regions might have arisen, not only from the different modalities that were used for the neuroimaging studies (functional neuroimaging studies ${ }^{1-13}$ versus a structural brain study ${ }^{14}$ ) but also, from the different analytical methods that were used (a region of interest approach ${ }^{12,13}$ versus a voxel-based analysis ${ }^{11,14}$ ).

Considering these results of previous neuroimaging studies together with the classification of agitated behaviors proposed by Cohen-Mansfield, ${ }^{3}$ we hypothesized that different dimensions of agitated behaviors are mediated by distinct neural systems. Two previous studies ${ }^{11,12}$ suggested that opposite hemisphere dominance (left ${ }^{11} \mathrm{vs} \mathrm{right}^{12}$ hemisphere) was responsible for the expression of agitated behaviors. Thus, whether a disruption of the right or left hemisphere dominance in the frontal and temporal regions is critical in different dimensions of agitated behaviors remains unclear.
We used SPECT to examine the relationships between regional cerebral blood flow (rCBF) and the presence of each dimension of agitated behavior in $\mathrm{AD}$ patients.

\section{Methods \\ Participants}

The patients were selected from a large group of our previous study, ${ }^{6}$ which enrolled 149 Japanese patients with AD who attended the outpatient clinic of Nagoya City University Hospital. The diagnostic evaluation included a complete history and physical examination, routine blood tests (including an evaluation of serum vitamin B12 and thyroid function), either a magnetic resonance imaging (MRI) or a computed tomography (CT) scan of the brain, and neuropsychological testing. The study inclusion criteria were: 1) a diagnosis of probable $\mathrm{AD}$ according to the National Institute of Neurological and Communication Disorders and Stroke/ Alzheimer's Disease and Related Disorders Association criteria; ${ }^{15} 2$ ) very mild to moderate (grade 0.5 , 1 , or 2 ) functional dementia severity on the clinical dementia rating scale (CDR); ${ }^{16}$ and 3) no history of taking medication containing acetylcholine esterase inhibitors or antipsychotic medications. Patients were excluded if: 1) other neurological diseases were present; 2) a previous history of mental illness or substance abuse prior to the onset of dementia was present; 3) either an MRI or a CT scan revealed focal brain lesions; 4) the Mini-Mental State Examination (MMSE ) ${ }^{17,18}$ score was less than 11; or 5) reliable informed consent could not be obtained from the patient and/or the patient's relatives.

The study protocol was approved by the Ethics Committee of Nagoya City University Medical School. Both the subjects and their caregivers were informed of the purpose and procedures of this study and signed a consent form.

\section{Psychiatric interview and diagnosis}

The diagnosis of agitated behaviors in the AD patients was assessed using the Japanese version of the ABID. ${ }^{6,19}$ The reliability and validity of the Japanese version of the test battery have been confirmed. ${ }^{6}$ Briefly, the Japanese version of the ABID demonstrated excellent internal reliability for both the frequency ratings (Cronbach's $\alpha=0.89$ ) and the reaction ratings (Cronbach's $\alpha=0.92$ ) and an excellent test-retest reliability for both the frequency ratings and the reaction ratings. The total score for the frequency ratings of the ABID was significantly associated with the CMAI.

The ABID includes items that can be observed and described objectively and that were identified by Logsdon et $\mathrm{a}^{19}$ as being the most problematic in individuals 
with dementia. It consists of a 16-item questionnaire seeking responses from caregivers about the common agitated behaviors in dementia patients. For the current study, the caregivers first rated each behavior according to the frequency of occurrence during each of the 2 weeks immediately before the assessment, using a scale of 0 to $3(0=$ did not occur during the week; $1=$ occurred one to two times during the week; $2=$ occurred three to six times during the week; $4=$ occurred daily or more often). The two weekly scores for each item were then added together; the resulting item scores ranged from 0 to 6 . The item scores were summed to obtain the total score, with possible scores ranging from 0 to 96 . In another section of the ABID, the caregivers were asked to rate their own reactions to each problem behavior, on a scale of 0 to 4 (where $0=$ not upsetting and $4=$ extremely upsetting). The caregiver's reactions are rated once for each item and are then summed. The total reaction scores have a possible range of 0 to 64 . The main aim of this study was to clarify the underlying mechanisms of the frequency of agitated behaviors in AD patients. Thus, we did not examine the neural basis of the caregiver's reactions because this assessment concerned the caregiver's level of distress.

Based on the results of a previous study, ${ }^{6}$ we obtained three factors in Japanese AD patients $(n=32)$. The detailed methods used for this analysis were previously reported. ${ }^{6}$ The current statistical analysis was performed as follows. A principal component factor analysis using Varimax rotation was performed for the 16 items included in the frequency rating of the ABID. The models included factors with an eigenvalue $>1$. An item was considered to load onto a factor if its factor-loading score exceeded 0.50. An exploratory principal component analysis with Varimax rotation using eigenvalues $>1$ reduced the 16 variables to three factors representing the frequency rating of the ABID. These three factors explained $70.2 \%$ of the variance in the frequency rating data of the ABID. A visual inspection of a scree plot also supported a three-factor solution. Table 1 demonstrates a rotated component matrix of the three-factor solution in Japanese AD patients $(n=32)$ in the current study. The first factor in the frequency rating data of the ABID had high loadings for items such as "physically threatening or aggressive toward others", "destroying property", and "restlessness, fidgetiness, inability to sit still". The first factor explained $41.8 \%$ of the variance. The first factor was termed "physically agitated behavior". The second factor in the frequency rating data of the ABID included most of the items corresponding to verbally agitated behavior, such as "arguing, irritability, or complaining" and "refusing to accept appropriate help". Therefore, we named this factor "verbally agitated behavior". The second factor explained $21.0 \%$ of the variance. The third factor in the frequency rating data of the ABID mainly contained items representing psychosis symptoms (delusion and hallucination), such as "seeing, hearing, or sensing distressing people or things that are not really present" and "incorrect, distressing beliefs". Therefore, the third factor was interpreted as representing "psychosis symptoms". The third factor explained $7.2 \%$ of the variance.

Thus, similar to our previous results, ${ }^{6}$ in the present study, we confirmed that three factors describing agitated behavior (frequency ratings) were identified using the Japanese

Table I Factor analysis of the ABID frequency scores among $32 \mathrm{AD}$ patients

\begin{tabular}{|c|c|c|c|c|}
\hline Item number & Agitated behavioral characteristics & Factor I & Factor 2 & Factor 3 \\
\hline 2 & Physically threatening or aggressive toward others & $0.953^{*}$ & 0.126 & 0.054 \\
\hline 5 & Destroying property & $0.917 *$ & 0.114 & 0.012 \\
\hline II & Restlessness, fidgetiness, and inability to sit still & $0.899 *$ & 0.157 & 0.056 \\
\hline 3 & Harmful to self & $0.837 *$ & 0.012 & 0.153 \\
\hline 7 & Trying to leave (or leaving) home inappropriately & $0.819 *$ & 0.219 & 0.215 \\
\hline 10 & Inappropriate sexual behavior & $0.710 *$ & 0.074 & 0.052 \\
\hline 8 & Arguing, irritability, or complaining & -0.099 & $0.894 *$ & 0.213 \\
\hline I & Verbally threatening or aggressive toward others & 0.042 & $0.843 *$ & 0.276 \\
\hline 4 & Inappropriate screaming or crying out & -0.085 & $0.766 *$ & 0.426 \\
\hline 13 & Easily agitated or upset & 0.389 & $0.748 *$ & 0.092 \\
\hline 12 & Worrying, anxiety, and/or fearfulness & 0.374 & $0.707^{*}$ & -0.050 \\
\hline 9 & Socially inappropriate behavior & 0.149 & $0.691 *$ & 0.318 \\
\hline 6 & Refusing to accept appropriate help & 0.422 & $0.553 *$ & 0.123 \\
\hline 16 & $\begin{array}{l}\text { Seeing, hearing, or sensing distressing people or things that are } \\
\text { not really present }\end{array}$ & 0.048 & 0.114 & $0.850 *$ \\
\hline 15 & Incorrect, distressing beliefs & 0.173 & 0.291 & $0.684^{*}$ \\
\hline 14 & Waking and getting up at night (other than trip to the bathroom) & 0.137 & 0.318 & $0.552^{*}$ \\
\hline
\end{tabular}

Note: *Factor loadings of 0.5 or more.

Abbreviations: ABID, Agitated Behavior in Dementia Scale; AD, Alzheimer disease. 
version of the ABID: factor 1) physically agitated behavior (eg, physically threatening or aggressive behavior toward others, destroying property, etc); factor 2) verbally agitated behavior (eg, arguing, irritability or complaining, and verbal threats); and factor 3) psychosis symptoms (eg, seeing, hearing or sensing distressing people or things that are not actually present, and incorrect or distressing beliefs).

\section{SPECT procedure}

Investigations using a SPECT scanner (e.cam ${ }^{\circledR}$; Siemens AG, Erlangen, Germany), to evaluate rCBF, were performed at the Department of Radiology of Nagoya City University Hospital. In AD patients with agitated behaviors, the SPECT scan was conducted within 1 month of the ABID assessment. All the patients were studied while lying in a supine resting position in a quiet room. The patients were instructed to remain awake with their eyes open and to lie still and not talk. A dose of approximately $600 \mathrm{MBq}$ of $99 \mathrm{mTc}-\mathrm{ECD}$ was injected intravenously. Fifteen minutes after the injection, the scans were performed using a dual-head gamma camera equipped with low-energy, high-resolution, parallel-hole collimators (e.cam ${ }^{\circledR}$; Siemens AG). The energy window was set at $140 \mathrm{keV}$ with a $20 \%$ width. The images were obtained using a $128 \times 128$ matrix and a pixel size of $3.3 \mathrm{~mm}$. The acquisition time for each projection was 20 seconds, with a total imaging time of 15 minutes for all 36 steps. Images were reconstructed using filtered back-projection (Ramp filters) with Butterworth prefiltering (power factor $=8$, cutoff frequency $=0.4$ cycles $/$ pixel). Attenuation correction was performed using Chang's algorithm, ${ }^{20}$ with an attenuation coefficient of $0.1 / \mathrm{cm}$. The spatial resolution at full width at half-maximum (FWHM) of the reconstructed images was $9 \mathrm{~mm}$.

\section{SPECT imaging analysis}

All the images were transferred from the SPECT imaging units to a Windows workstation (Windows 7, Microsoft Corporation, Redmond, WA, USA), where statistical analyses of all the data were conducted on a voxel-by-voxel basis, using statistical parametric mapping (SPM) software (SPM8; Wellcome Trust Centre for Neuroimaging, University College London, London, UK) implemented in MATLAB 7.5 (The MathWorks, Inc, Natick, MA, USA). Using a 99mTc-ECD template, the SPECT data were normalized into a standard stereotaxic space at the MNI (Montreal Neurological Institute) and smoothed using a $12 \mathrm{~mm}$ isotropic Gaussian filter. For SPECT analyses, an isotropic Gaussian filter is recommended for use at the FWHM of the SPECT device. ${ }^{21}$ In most previous SPECT studies, the images were smoothed using a $12 \mathrm{~mm}$ Gaussian filter. ${ }^{22,23}$ Thus, we utilized a $12 \mathrm{~mm}$ isotropic Gaussian filter to smooth the images. We used the adjusted $\mathrm{rCBF}$ in the relative flow distribution (normalization of global cerebral blood flow for each patient to $50 \mathrm{~mL} / 100 \mathrm{~g} / \mathrm{min}$, with proportional scaling) because the absolute rCBF is susceptible to general arousal, aging, and drug effects. ${ }^{24}$

We examined the relationship between the factor scores of the frequency ratings for each of the three subtypes of agitation (physically agitated behavior, verbally agitated behavior, and psychosis symptoms), identified using a factor analysis of ABID results, ${ }^{6}$ and the $\mathrm{rCBF}$ with covariate-only design matrices, using a multiple regression model implemented in SPM8. Age, sex, years of education, duration of illness, and the MMSE score were treated as nuisance covariates. The statistical significance level was the threshold for the correction of multiple comparisons using a false discovery rate (FDR) ${ }^{25}$ of 0.05 . Cluster extent thresholds were set at 100 voxels to reduce possible noise, based on previous studies. ${ }^{26,27}$ The cluster locations were converted from coordinates related to the MNI atlas system $^{28}$ to coordinates related to the Talairach atlas. ${ }^{29}$

\section{Results}

\section{Demographic and clinical characteristics}

Among 149 Japanese patients with AD, 40 AD patients could not undergo a SPECT examination. We excluded an additional $77 \mathrm{AD}$ patients because these patients did not meet the inclusion criteria, for reasons such as the use of antipsychotic medications. Thus, we analyzed the remaining 32 AD patients.

Table 2 shows the mean scores and standard deviations of the clinical and demographic characteristics of the $32 \mathrm{AD}$ patients. All 32 AD patients were right-handed patients. The ABID frequency ratings and reaction ratings were relatively high. For the CDR, no significant differences in the ABID frequency ratings were seen among the three groups in the AD patients (CDR 0.5: 40.0 \pm , CDR 1: 42.3 \pm 10.3 , and CDR 2: 42.7 \pm 5.3$)$.

\section{SPECT results}

The multiple regression analysis showed a negative correlation between the factor scores of the frequency rating of ABID representing physically agitated behavior (factor 1), and the right superior temporal gyrus (Brodmann 22) and the right inferior frontal gyrus (Brodmann 47$)\left(P_{\text {FDR-CORR }} 0.05\right)$ (Table 3 and Figure 1). No significant positive correlations between the factor scores of the frequency rating of ABID 
Table 2 The clinical and demographic data of $A D$ patients

\begin{tabular}{ll}
\hline Demographic variables & $\begin{array}{l}\text { AD patients } \\
(\mathbf{n}=\mathbf{3 2})\end{array}$ \\
\hline Age, years & $73.3 \pm 8.1$ \\
Education, years & $9.8 \pm 0.7$ \\
Sex (males/females) & $10 / 22$ \\
Dementia history & \\
$\quad$ Duration of illness, years & $3.9 \pm 1.2$ \\
MMSE & $18.2 \pm 2.9$ \\
CDR (0.5/I/2) & $2 / 20 / 10$ \\
ABID questionnaire & \\
Frequency ratings & $33.8 \pm 11.3$ \\
Mean factor score (ABID: frequency ratings) & \\
Factor I (physically agitated behavior) & $0.2025 \pm 0.9967$ \\
Factor 2 (verbally agitated behavior) & $0.3608 \pm 0.9177$ \\
Factor 3 (psychosis symptoms) & $0.2586 \pm 1.2560$ \\
\hline
\end{tabular}

Note: Data are presented as the mean \pm SD.

Abbreviations: ABID, Agitated Behavior in Dementia Scale; AD, Alzheimer disease; CDR, Clinical Dementia Rating; MMSE, Mini-Mental State Examination; SD, standard deviation.

representing physically agitated behavior (factor 1), and the rCBF were observed.

Regarding the factor 2 symptoms, representing verbally agitated behavior, the multiple regression analysis showed a negative correlation between the factor 2 scores of the frequency rating of $\mathrm{ABID}$ and the left inferior frontal gyrus (Brodmann 46), the left insula (Brodmann 13), and the left inferior frontal gyrus (Brodmann 44) $\left(P_{\text {FDR-CORR }} 0.05\right)$ (Table 4 and Figure 2). No significant positive correlations between the factor scores of the frequency rating of ABID representing verbally agitated behavior (factor 2), and the rCBF were observed.

Regarding the factor 3 symptoms, representing psychosis symptoms, the multiple regression analysis showed a negative correlation between the factor scores of the frequency rating of ABID and the right angular gyrus (right parietal lobe) (Brodmann 39) and the right occipital lobe (Brodmann19) $\left(P_{\text {FDR-CORR }} 0.05\right)$ (Table 5 and Figure 3$)$. No significant positive correlations between the factor scores of the frequency rating of ABID representing psychosis symptoms (factor 3), and the $\mathrm{rCBF}$ were observed.

\section{Discussion}

To our knowledge, this is the first study to examine different dimensions of agitated behaviors using SPECT, in AD patients. We identified three different dimensions of the ABID that were associated with distinct neural areas of $\mathrm{rCBF}$ in $\mathrm{AD}$ patients. Firstly, the result of the analysis of the factor 1 symptoms suggests that lower rCBF values in the right superior temporal gyrus (Brodmann 22) and the right inferior frontal gyrus (Brodmann 47) were correlated with an increase in the score for ABID factor 1 symptoms. Secondly, the result of analysis of the factor 2 symptoms suggests that lower rCBF values in the left inferior frontal gyrus (Brodmann 46), the left insula (Brodmann 13), and the left inferior frontal gyrus (Brodmann 44) were correlated with an increase in the score for ABID factor 2 symptoms.

Thirdly, the result of the analysis of the factor 3 symptoms suggests that lower rCBF values in the right angular gyrus (Brodmann 39) and the right occipital lobe (Brodmann 19) were correlated with an increase in the score for ABID factor 3 symptoms.

Dysfunction in the right hemisphere is known to produce marked emotionally relevant abnormalities and the disruption of social behavior. Many studies have reported a wide variety of inappropriate agitated behaviors, such as mania and hypersexual activity, to be associated with right hemisphere brain lesions. ${ }^{30,31}$ A previous study ${ }^{12}$ of $\mathrm{AD}$ patients supported a right hemisphere dominance in the regulation of agitated behaviors. In our study, one of the ABID dimensions (physically agitated behavior) supports the hypothesis that the right hemisphere is dominant for the regulation of agitated behaviors. We observed that two areas, including both the right superior temporal and inferior frontal lobes, are associated with physically agitated behavior. Three previous studies have suggested that AD patients with aggression exhibit hypoperfusion in the temporal lobe. ${ }^{11-13}$ However, both the parts and the laterality of the temporal lobe related to agitated behaviors differed among the three studies. These differences might be partly attributable to variations in the

Table 3 Significant negative correlations between $\mathrm{rCBF}$ values and the factor scores of the frequency rating of the ABID in relation to physically agitated behavior, for all AD patients

\begin{tabular}{|c|c|c|c|c|c|c|c|}
\hline \multirow[t]{2}{*}{ Cluster } & \multirow[t]{2}{*}{ Region } & \multirow{2}{*}{$\begin{array}{l}\text { Brodmann's } \\
\text { area }\end{array}$} & \multicolumn{3}{|c|}{ Talairach coordinates } & \multirow{2}{*}{$\begin{array}{l}\text { Voxels in } \\
\text { cluster }\end{array}$} & \multirow[t]{2}{*}{$Z$-value } \\
\hline & & & $x$ & $y$ & $\mathbf{z}$ & & \\
\hline 1 & Rt superior temporal gyrus & 22 & 63 & -7 & -1 & 373 & 3.61 \\
\hline 2 & Rt inferior frontal gyrus & 47 & 16 & 10 & -21 & 132 & 3.68 \\
\hline 3 & Rt inferior frontal gyrus & 47 & 27 & 9 & -15 & 132 & 3.45 \\
\hline
\end{tabular}

Notes: $\mathrm{N}=32 . P_{\text {FDR-CORR }} 0.05$ (results of a voxel-wise SPM analysis).

Abbreviations: ABID, Agitated Behavior in Dementia Scale; AD, Alzheimer disease; CORR, correction; FDR, false discovery rate; rCBF, regional cerebral blood flow; Rt, right; SPM, statistical parametric mapping. 

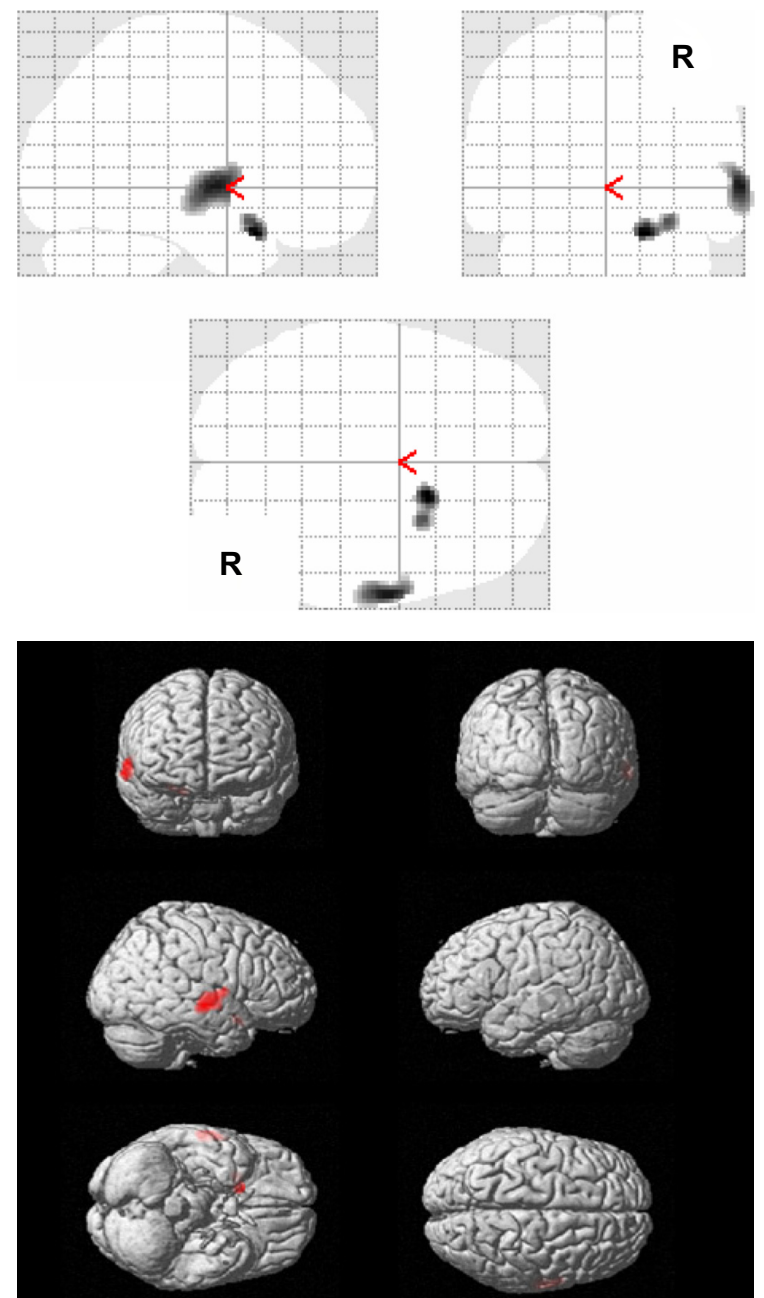

Figure I Results of SPM analysis in relation to physically agitated behavior. Notes: The figure shows areas with significant negative correlations between the $\mathrm{rCBF}$ values and the factor scores of the frequency rating of the ABID in relation to physically agitated behavior, for all AD patients $(n=32)\left(P_{\text {FDR-CORR }} 0.05\right)$. The Talairach coordinates are given in Table 3.

Abbreviations: ABID, Agitated Behavior in Dementia Scale; AD, Alzheimer disease; CORR, correction; FDR, false discovery rate; $r C B F$, regional cerebral blood flow; SPM, statistical parametric mapping.

methods used to assess agitation in dementia. Unlike a multisymptom scale, such as the Neuropsychiatric Inventory (NPI), ${ }^{32}$ the ABID was devised to assess specific agitated behaviors in dementia patients. Several items in the factor termed as physically agitated behavior according to the ABID (eg, restlessness, trying to leave, and inappropriate sexual behavior) correspond to the items of disinhibition or aberrant motor behavior, according to the NPI or other assessments. Zamboni et $\mathrm{al}^{133}$ showed that the severity of disinhibition in patients with frontotemporal dementia was correlated with gray matter loss in the right lateral temporal lobe (middle temporal gyrus). In addition, Mendez et al ${ }^{34}$ suggested that right temporal involvement may cause emotional impairments in frontotemporal dementia. Thus, the dysfunction of the right side of the temporal lobe may contribute to the expression of physically agitated behaviors, not only in patients with frontotemporal dementia but also, in $\mathrm{AD}$ patients.

Another area, the right inferior frontal gyrus (area 47) is located in the lateral orbitofrontal cortex (OFC). Several neuroimaging studies of dementia patients have implicated the involvement of the OFC in behavioral disturbances resembling the physically agitated behaviors observed in the present study. ${ }^{35-37}$ A histopathological study has suggested a relationship between neurofibrillary tangles in the OFC and the severity of agitation in AD patients. ${ }^{38}$ Patients with focal damage in the OFC are known to have poor social judgment and to exhibit socially inappropriate behavior. ${ }^{39,40}$ Damage to the OFC is believed to result in an inability to suppress negative emotion, as a core factor in aggression. ${ }^{41}$ Recently, functional distinctions between the medial and lateral OFC have become a matter of controversy. In contrast to the medial $\mathrm{OFC}$, the lateral OFC (more specifically, the right lateral $\mathrm{OFC}$ ) has been suggested to play a role in regulating angry emotional responses to others and in evaluating punishments, so as to alter behavior. ${ }^{42}$

Taken together with the above findings, hypoperfusion in both the right superior temporal gyrus (Brodmann 22) and the inferior frontal gyrus (Brodmann 47) may contribute to disruptions in the ability of AD patients to control emotional states associated with physically agitated behavior.

In contrast to physically agitated behavior, the left hemisphere was shown to be involved in verbally agitated behavior. Manifestations of verbally agitated behavior are thought to arise as a result of social isolation, caused by

Table 4 Significant negative correlations between $\mathrm{rCBF}$ values and the factor scores of the frequency rating of the ABID in relation to verbally agitated behavior, for all $A D$ patients

\begin{tabular}{|c|c|c|c|c|c|c|c|}
\hline \multirow[t]{2}{*}{ Cluster } & \multirow[t]{2}{*}{ Region } & \multirow{2}{*}{$\begin{array}{l}\text { Brodmann's } \\
\text { area }\end{array}$} & \multicolumn{3}{|c|}{ Talairach coordinates } & \multirow{2}{*}{$\begin{array}{l}\text { Voxels in } \\
\text { cluster }\end{array}$} & \multirow[t]{2}{*}{$Z$-value } \\
\hline & & & $\mathbf{x}$ & $y$ & $\bar{z}$ & & \\
\hline I & Lt inferior frontal gyrus & 46 & -53 & 34 & 14 & 500 & 4.71 \\
\hline 2 & Lt insula & 13 & -35 & 13 & -2 & 388 & 3.88 \\
\hline 3 & Lt inferior frontal gyrus & 44 & -49 & 10 & 16 & 388 & 3.50 \\
\hline
\end{tabular}

Notes: $\mathrm{N}=32$. $P_{\text {FDR-CORR }} 0.05$ (results of a voxel-wise SPM analysis).

Abbreviations: ABID, Agitated Behavior in Dementia Scale; AD, Alzheimer disease; CORR, correction; FDR, false discovery rate; Lt, left; rCBF, regional cerebral blood flow; SPM, statistical parametric mapping. 

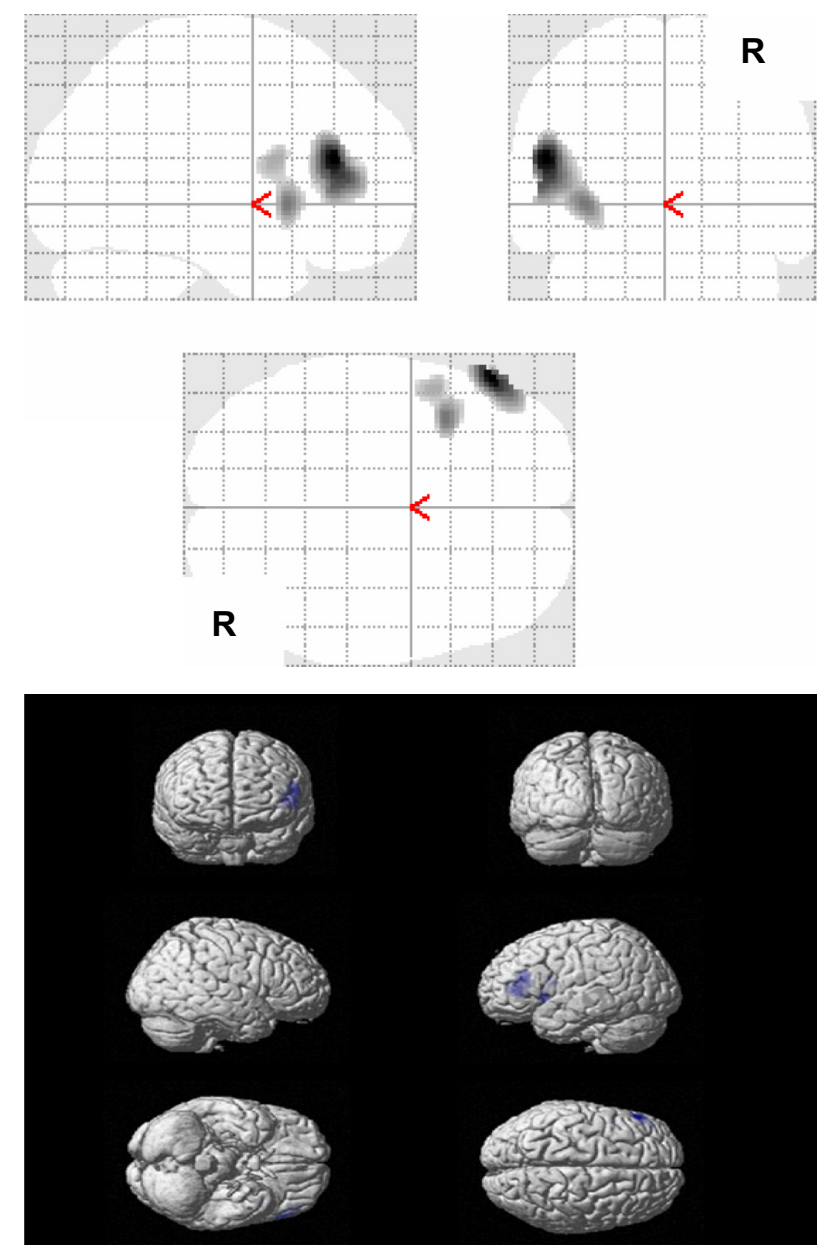

Figure 2 Results of SPM analysis in relation to verbally agitated behavior. Notes: The figure shows areas with significant negative correlations between the $\mathrm{rCBF}$ values and the factor scores of the frequency rating of the ABID in relation to verbally agitated behavior, for all $A D$ patients $(n=32)\left(P_{\text {FDR-CORR }} 0.05\right)$.

Abbreviations: ABID, Agitated Behavior in Dementia Scale; AD, Alzheimer disease; CORR, correction; FDR, false discovery rate; $r C B F$, regional cerebral blood flow; SPM, statistical parametric mapping.

poor communication abilities. ${ }^{3,43}$ Verbally agitated behavior, such as inappropriate screaming, is likely to be associated with specific requests or undefined needs, including calls for attention, because $\mathrm{AD}$ patients with declining language abilities cannot adequately express either discomfort or pain to their caregivers., ${ }^{3,43}$ Thus, various verbally agitated behaviors may be produced by the dysfunction of the left hemisphere mediating verbal skills and verbal semantic memory. ${ }^{44}$ We observed a mainly lower rCBF in the left lateral inferior frontal lobe (Brodmann 44, 46) in association with verbally agitated behavior. Hirono et $\mathrm{al}^{11}$ hypothesized that the dysfunction of the dorsolateral frontal lobes may cause misinterpretations of the environment, resulting in aggressive behaviors. Many studies using neuroimaging study have revealed that the left dorsolateral frontal cortex (particularly the left inferior frontal gyrus) is necessary for the control of switching and inhibitory behaviors. ${ }^{45,46}$ Recently, a PET study by Schroeter et $\mathrm{a}^{47}$ revealed that the left lateral inferior frontal area underlies executive deficits in dementia patients. Therefore, the lower rCBF in the left lateral inferior frontal area may cause executive deficits associated with verbal skills, contributing to verbally agitated behavior. We also observed that a significantly lower rCBF in the left insula was associated with verbally agitated behavior. Consistent with our findings, Bruen et $\mathrm{al}^{14}$ identified an association between left insular damage and agitation in $\mathrm{AD}$ patients. The insula is critical for the expression of pain and the integration of internal and external stimuli. ${ }^{14,27}$ Dementia patients suffering from verbally agitated behavior have higher levels of both pain and discomfort. ${ }^{3,43}$ Thus, the lower rCBF in the left insula may cause several unpleasant feelings, such as pain and anxiety, resulting in specific verbal requests.

The factor 3 symptoms, representing psychotic symptoms, include delusions (incorrect or distressing beliefs), hallucination (seeing, hearing, or sensing distressing people or things that are not really present), and wandering (waking and getting up at night, other than for trips to the bathroom). Unlike the ABID, the CMAI does not include any items associated with either delusions or hallucinations. The subtypes of agitated behavior proposed by Cohen-Mansfield ${ }^{3}$ do not include either delusions or hallucinations. However, delusion, hallucination, and wandering are well known to result in agitated behaviors because of inappropriate internal stimuli. ${ }^{1,8}$ Thus, psychotic symptoms can be regarded as one type of agitated behavior in dementia patients.

Two previous studies have demonstrated that right posterior parietal hypoperfusion is correlated with delusion in $\mathrm{AD}$

Table 5 Significant negative correlations between $\mathrm{rCBF}$ values and the factor scores of the frequency rating of the ABID in relation to psychosis symptoms, for all AD patients

\begin{tabular}{|c|c|c|c|c|c|c|c|}
\hline \multirow[t]{2}{*}{ Cluster } & \multirow[t]{2}{*}{ Region } & \multirow{2}{*}{$\begin{array}{l}\text { Brodmann's } \\
\text { area }\end{array}$} & \multicolumn{3}{|c|}{ Talairach coordinates } & \multirow{2}{*}{$\begin{array}{l}\text { Voxels in } \\
\text { cluster }\end{array}$} & \multirow[t]{2}{*}{ Z-value } \\
\hline & & & $x$ & $y$ & $\mathbf{z}$ & & \\
\hline 1 & Rt angular gyrus & 39 & 38 & -65 & 20 & 505 & 5.21 \\
\hline 2 & Rt occipital lobe & 19 & 30 & -77 & 35 & 505 & 5.16 \\
\hline
\end{tabular}

Notes: $\mathrm{N}=32$. $P_{\text {FDR-CORR }} 0.05$ (results of a voxel-wise SPM analysis).

Abbreviations: ABID, Agitated Behavior in Dementia Scale; AD, Alzheimer disease; CORR, correction; FDR, false discovery rate; rCBF, regional cerebral blood flow; Rt, right; SPM, statistical parametric mapping. 

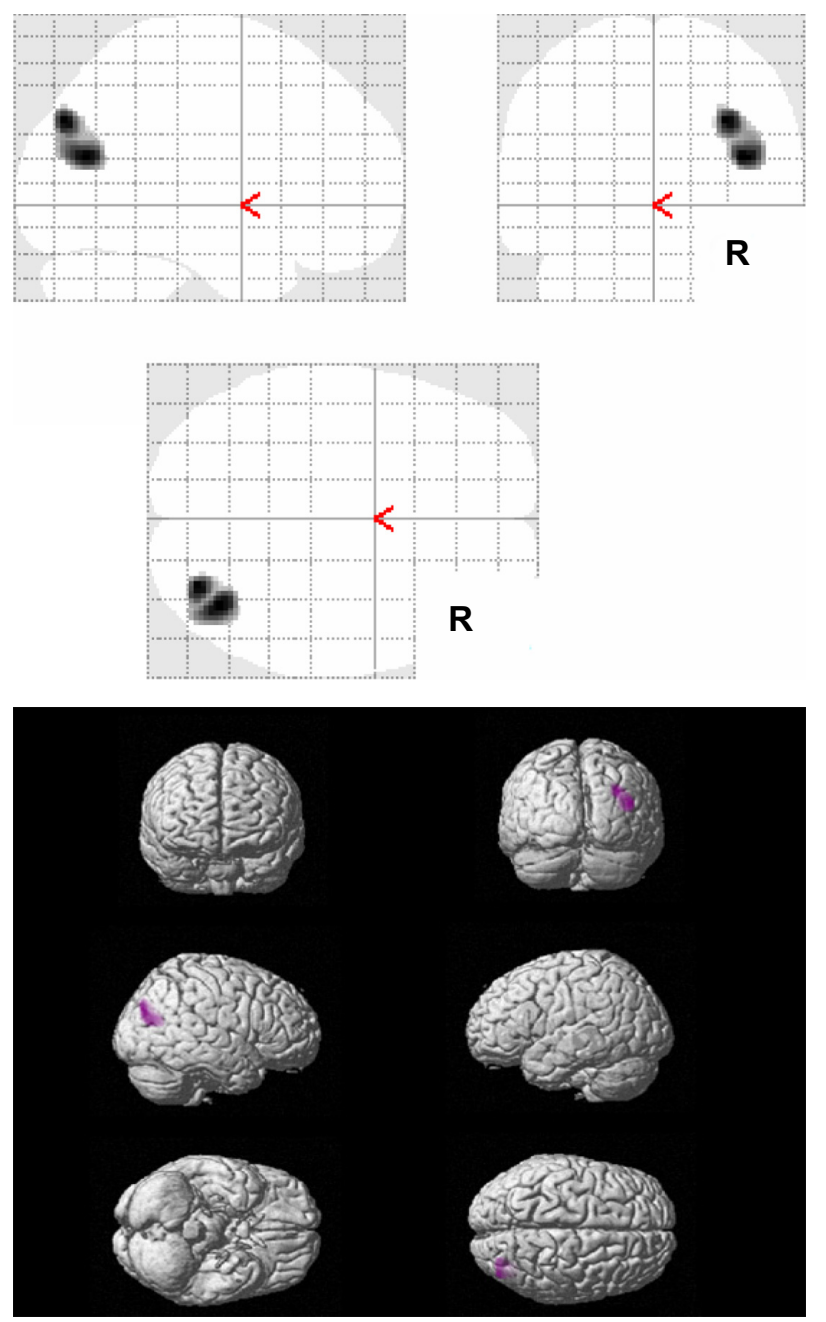

Figure 3 Results of SPM analysis in relation to psychosis symptoms.

Notes: The figure shows areas with significant negative correlations between the $\mathrm{rCBF}$ values and the factor scores of the frequency rating of the $A B I D$ in relation to psychosis symptoms, for all $A D$ patients $(n=32)\left(P_{\text {FDR-CORR }} 0.05\right)$.

Abbreviations: $A B I D$, Agitated Behavior in Dementia Scale; $A D$, Alzheimer disease; FDR, false discovery rate; rCBF, regional cerebral blood flow; SPM, statistical parametric mapping.

patients. ${ }^{26,48}$ Hallucination in AD patients was also found to be associated with hypoperfusion in the right parietal lobe. ${ }^{49,50}$ Parieto-occipital lesions, in dementia associated with Lewy body disease, have been implicated in the pathology of visual hallucination. ${ }^{51}$ In a SPECT study in AD patients, Rolland et $\mathrm{al}^{52}$ revealed that hypoperfusion in the left parietotemporal area was associated with wandering. In addition, a right hemispheric pathology is believed to contribute to the expression of delusions in dementia. ${ }^{35,53}$ Our findings support the hypothesis that right parieto-occipital dysfunction may be responsible for psychotic symptoms in AD patients because of deficits in both attention and visual systems. ${ }^{48,51}$ However, our study could not find any abnormalities in the frontal lobe corresponding to psychotic factors. Recent studies using neuroimaging have shown that the right side of either the frontal or temporal lobe is critical for delusions in AD patients. ${ }^{14,54,55}$ A possible reason is that the limited types of questions for delusions may not be sufficient to analyze the neural basis underlying various types of delusions, such as misidentification delusions.

Finally, we must address several limitations of the present study. Firstly, we used a normalized global cerebral blood flow of $50 \mathrm{~mL} / 100 \mathrm{~g} / \mathrm{min}$, with proportional scaling for each patient, in the SPM analysis. However, the average blood flow in the whole brain may be relatively low in AD patients; accordingly, some rCBF reductions in certain areas might not have been detected in the AD patients. ${ }^{56-58}$ Thus, the use of either cerebellar or sensorimotor cortical normalization, which are not affected by pathological involvement in AD patients, might be more useful for accurately identifying hypoperfusion associated with agitated behaviors in AD patients. In future studies, SPECT investigations of agitated behaviors in AD patients that are performed using SPM analyses should be assessed using reliable reference regions, such as either the cerebellar or sensorimotor cortical cortex.

Secondly, we did not examine AD patients with severe cognitive impairments because the ABID was designed for use in patients with mild to moderate levels of dementia. However, it is important to note that even mild or moderate levels of dementia at the time of the first assessment may progress to a more severe stage during the follow-up period, as the disease progresses. Thus, a future study may be required to use the CMAI to examine the neural basis of the agitated behaviors occurring with more advanced AD.

Thirdly, we could not assess the ABID on the day of the SPECT examinations. Although the SPECT scan was conducted within 1 month of the ABID assessment, our study may reflect trait-related agitated behaviors in AD patients. Fourthly, apart from the MMSE, we did not assess other cognitive tasks, such as executive function or language tasks. Thus, the association between these cognitive functions and different agitated behaviors in AD patients remains unclear. Also, we could not confirm a wide variety of behavior symptoms because we did not utilize a multisymptom rating scale, such as the NPI. ${ }^{30}$ Finally, our study did not include either an age-matched normal elderly control group or patients with other types of dementia. Thus, we could not confirm whether the observed findings in this study were a specific result of $\mathrm{AD}$ alone.

Despite these limitations, our study using SPECT confirmed that different dimensions of agitated behaviors in AD patients are mediated by distinct neural systems. This study 
adds to important neuroimaging evidence regarding the neural basis of behavior disorders in dementia patients. The findings of the present study may contribute, not only to a deeper understating of the neural basis of agitated behaviors but also, to the formulation of more effective individualized treatment plans and management of agitated behaviors in dementia.

\section{Acknowledgments}

The authors gratefully acknowledge a Grant-in-Aid for Scientific Research (grant number: 25461782) from the Ministry of Education, Culture, Sports, Sciences, and Technology in Japan.

\section{Disclosure}

The authors report no conflict of interest in this work.

\section{References}

1. Mega MS, Cummings JL, Fiorello T, Gornbein J. The spectrum of behavioral changes in Alzheimer's disease. Neurology. 1996;46(1): 130-135.

2. Hirono N, Mori E, Tanimukai S, et al. Distinctive neurobehavioral features among neurodegenerative dementias. $J$ Neuropsychiatry Clin Neurosci. 1999;11(4):498-503.

3. Cohen-Mansfield J. Agitation in the elderly definitional and theoretical conceptualizations. In: Hay DP, Klein DT, Hay LK, Grossberg GT, Kennedy JS, editors. Agitation in Patients with Dementia: A Practical Guide to Diagnosis and Management. Washington, DC: American Psychiatric Publishing Inc.; 2003:1-21.

4. Cohen-Mansfield J, Marx MS, Rosenthal AS. A description of agitation in a nursing home. $J$ Gerontol. 1989;44(3):M77-M84.

5. Rabinowitz J, Davidson M, De Deyn PP, Katz I, Brodaty H, Cohen-Mansfield J. Factor analysis of the Cohen-Mansfield Agitation Inventory in three large samples of nursing home patients with dementia and behavioral disturbance. Am J Geriatr Psychiatry. 2005;13(11): 991-998.

6. Torii K, Nakaaki S, Banno K, et al. Reliability and validity of the Japanese version of the Agitated Behaviour in Dementia Scale in Alzheimer's disease: three dimensions of agitated behaviour in dementia. Psychogeriatrics. 2011;11(4):212-220.

7. Cohen-Mansfield J, Werner P, Reisberg B. Temporal order of cognitive and functional loss in a nursing home population. $J$ Am Geriatr Soc. 1995;43(9):974-978.

8. Cohen-Mansfield J, Werner P. Longitudinal changes in behavioral problems in old age: a study in an adult day care population. $J$ Gerontol A Biol Sci Med Sci. 1998;53(1):M65-M71.

9. Chen ST, Sultzer DL, Hinkin CH, Mahler ME, Cummings JL. Executive dysfunction in Alzheimer's disease: association with neuropsychiatric symptoms and functional impairment. J Neuropsychiatry Clin Neurosci. 1998;10(4):426-432.

10. Senanarong V, Cummings JL, Fairbanks L, et al. Agitation in Alzheimer's disease is a manifestation of frontal lobe dysfunction. Dement Geriatr Cogn Disord. 2004;17(1-2):14-20.

11. Hirono N, Mega MS, Dinov ID, Mishkin F, Cummings JL. Left frontotemporal hypoperfusion is associated with aggression in patients with dementia. Arch Neurol. 2000;57(6):861-866.

12. Lanctôt KL, Herrmann N, Nadkarni NK, Leibovitch FS, Caldwell CB, Black SE. Medial temporal hypoperfusion and aggression in Alzheimer disease. Arch Neurol. 2004;61(11):1731-1737.
13. Sultzer DL, Mahler ME, Mandelkern MA, et al. The relationship between psychiatric symptoms and regional cortical metabolism in Alzheimer's disease. J Neuropsychiatry Clin Neurosci. 1995;7(4):476-484.

14. Bruen PD, McGeown WJ, Shanks MF, Venneri A. Neuroanatomical correlates of neuropsychiatric symptoms in Alzheimer's disease. Brain. 2008;131(Pt 9):2455-2463.

15. McKhann G, Drachman D, Folstein M, Katzman R, Price D, Stadlan EM. Clinical diagnosis of Alzheimer's disease: report of the NINCDS-ADRDA Work Group under the auspices of Department of Health and Human Services Task Force on Alzheimer's Disease. Neurology. 1984;34(7):939-944.

16. Hughes CP, Berg L, Danziger WL, Coben LA, Martin RL. A new clinical scale for the staging of dementia. Br J Psychiatry. 1982;140: 566-572.

17. Folstein MF, Folstein SE, McHugh PR. "Mini-mental state". A practical method for grading the cognitive state of patients for the clinician. J Psychiatr Res. 1975;12(3):189-198.

18. Sugishita M. [Mini Mental State Examination-Japanese]. Tokyo: Nihon Bunka Kagakusha Company; 2012. Japanese.

19. Logsdon RG, Teri L, Weiner MF, et al. Assessment of agitation in Alzheimer's disease: the agitated behavior in dementia scale. Alzheimer's Disease Cooperative Study. J Am Geriatr Soc. 1999;47(11): 1354-1358.

20. Chang LT. A method for attenuation correction in radionuclide computed tomography. IEEE Trans Nucl Sci. 1978;25(1):638-643. Japanese.

21. Ohnishi T. SPM. In: Matsuda H, Asada T, editors. Imaging Diagnosis for Dementia. Osaka: Nagai Shoten; 2004:73-89.

22. Matsuda H, Ohnishi T, Asada T, et al. Correction for partial-volume effects on brain perfusion SPECT in healthy men. J Nucl Med. 2003;44(8):1243-1252.

23. Matsuda H, Mizumura S, Soma T, Takemura N. Conversion of brain SPECT images between different collimators and reconstruction processes for analysis using statistical parametric mapping. Nucl Med Commun. 2004;25(1):67-74.

24. Imon Y, Matsuda H, Ogawa M, Kogure D, Sunohara N. SPECT image analysis using statistical parametric mapping in patients with Parkinson's disease. J Nucl Med. 1999;40(10):1583-1589.

25. Genovese CR, Lazar NA, Nichols T. Thresholding of statistical maps in functional neuroimaging using the false discovery rate. Neuroimage. 2002;15(4):870-878.

26. Nomura K, Kazui H, Wada T, et al. Classification of delusions in Alzheimer's disease and their neural correlates. Psychogeriatrics. 2012;12(3):200-210.

27. Matsuoka T, Narumoto J, Shibata K, et al. Insular hypoperfusion correlates with the severity of delusions in individuals with Alzheimer's disease. Dement Geriatr Cogn Disord. 2010;29(4):287-293.

28. Petrides M. The Human Cerebral Cortex: An MRIAtlas of the Sulci and Gyri in MNI Stereotaxic Space. New York: Academic Press; 2011.

29. Talairach J. Co-Planar Stereotaxic Atlas of the Human Brain: 3-Dimensional Proportional System : An Approach to Cerebral Imaging (Thieme Classics). New York: George Thieme Verlag; 1988.

30. Cummings JL, Mendez MF. Secondary mania with focal cerebrovascular lesions. Am J Psychiatry. 1984;141(9):1084-1087.

31. Cummings JL. Neuropsychiatric manifestations of right hemisphere lesions. Brain Lang. 1997;57(1):22-37.

32. Cummings JL, Mega M, Gray K, Rosenberg-Thompson S, Carusi DA, Gornbein J. The Neuropsychiatric Inventory: comprehensive assessment of psychopathology in dementia. Neurology. 1994;44(12): 2308-2314.

33. Zamboni G, Huey ED, Krueger F, Nichelli PF, Grafman J. Apathy and disinhibition in frontotemporal dementia: Insights into their neural correlates. Neurology. 2008;71(10):736-742.

34. Mendez MF, McMurtray A, Chen AK, Shapira JS, Mishkin F, Miller BL. Functional neuroimaging and presenting psychiatric features in frontotemporal dementia. J Neurol Neurosurg Psychiatry. 2006;77(1):4-7.

35. Rosen HJ, Allison SC, Schauer GF, Gorno-Tempini ML, Weiner MW, Miller BL. Neuroanatomical correlates of behavioural disorders in dementia. Brain. 2005;128(Pt 11):2612-2625. 
36. Massimo L, Powers C, Moore P, et al. Neuroanatomy of apathy and disinhibition in frontotemporal lobar degeneration. Dement Geriatr Cogn Disord. 2009;27(1):96-104.

37. Reilly TJ, Staff RT, Ahearn TS, Bentham P, Wischik CM, Murray AD. Regional cerebral blood flow and aberrant motor behaviour in Alzheimer's disease. Behav Brain Res. 2011;222(2):375-379.

38. Tekin S, Mega MS, Masterman DM, et al. Orbitofrontal and anterior cingulate cortex neurofibrillary tangle burden is associated with agitation in Alzheimer disease. Ann Neurol. 2001;49(3):355-361.

39. Eslinger PJ, Damasio AR. Severe disturbance of higher cognition after bilateral frontal lobe ablation: patient EVR. Neurology. 1985;35(12):1731-1741.

40. Anderson SW, Bechara A, Damasio H, Tranel D, Damasio AR. Impairment of social and moral behavior related to early damage in human prefrontal cortex. Nat Neurosci. 1999;2(11):1032-1037.

41. Davidson RJ, Putnam KM, Larson CL. Dysfunction in the neural circuitry of emotion regulation - a possible prelude to violence. Science. 2000;289(5479):591-594.

42. Hooker CI, Knight RT. The role of lateral orbitofrontal cortex in the inhibitory control of emotion. In: Zald DH, Rauch SL, editors. The Orbitofrontal Cortex. New York, NY: Oxford University Press; 2006:307-324.

43. Cohen-Mansfield J, Werner P. Typology of disruptive vocalizations in older persons suffering from dementia. Int J Geriatr Psychiatry. 1997;12(11):1079-1091.

44. Hirono N, Mori E, Ishii K, et al. Neuronal substrates for semantic memory: a positron emission tomography study in Alzheimer's disease. Dement Geriatr Cogn Disord. 2001;12(1):15-21.

45. Nagahama Y, Fukuyama $H$, Yamauchi $H$, et al. Cerebral activation during performance of a card sorting test. Brain. 1996;119(Pt 5):1667-1675.

46. Zakzanis KK, Mraz R, Graham SJ. An fMRI study of the Trail Making Test. Neuropsychologia. 2005;43(13):1878-1886.

47. Schroeter ML, Vogt B, Frisch S, et al. Executive deficits are related to the inferior frontal junction in early dementia. Brain. 2012;135(Pt 1): 201-215.

48. Fukuhara R, Ikeda M, Nebu A, et al. Alteration of rCBF in Alzheimer's disease patients with delusions of theft. Neuroreport. 2001;12(11): 2473-2476.
49. Kotrla KJ, Chacko RC, Harper RG, Jhingran S, Doody R. SPECT findings on psychosis in Alzheimer's disease. Am J Psychiatry. 1995;152(10):1470-1475.

50. Lopez OL, Smith G, Becker JT, Meltzer CC, DeKosky ST. The psychotic phenomenon in probable Alzheimer's disease: a positron emission tomography study. J Neuropsychiatry Clin Neurosci. 2001;13(1):50-55.

51. Nagahama Y, Okina T, Suzuki N, Matsuda M. Neural correlates of psychotic symptoms in dementia with Lewy bodies. Brain. 2010; 133(Pt 2):557-567.

52. Rolland Y, Payoux P, Lauwers-Cances V, Voisin T, Esquerré JP, Vellas B. A SPECT study of wandering behavior in Alzheimer's disease. Int $J$ Geriatr Psychiatry. 2005;20(9):816-820.

53. Ismail Z, Nguyen MQ, Fischer CE, Schweizer TA, Mulsant BH. Neuroimaging of delusions in Alzheimer's disease. Psychiatry Res. 2012;202(2):89-95.

54. Nakano S, Yamashita F, Matsuda H, Kodama C, Yamada T. Relationship between delusions and regional cerebral blood flow in Alzheimer's disease. Dement Geriatr Cogn Disord. 2006;21(1):16-21.

55. Lee E, Kinomura S, Meguro K, Akanuma K, Meguro M, Fukuda H. Confabulations on episodic and semantic memory questions are associated with different neurologic backgrounds in Alzheimer disease. Cogn Behav Neurol. 2009;22(2):81-88.

56. Soonawala D, Amin T, Ebmeier KP, et al. Statistical parametric mapping of (99m)Tc-HMPAO-SPECT images for the diagnosis of Alzheimer's disease: normalizing to cerebellar tracer uptake. Neuroimage. 2002;17(3):1193-1202.

57. Yakushev I, Hammers A, Fellgiebel A, et al. SPM-based count normalization provides excellent discrimination of mild Alzheimer's disease and amnestic mild cognitive impairment from healthy aging. Neuroimage. 2009;44(1):43-50.

58. Küntzelmann A, Guenther T, Haberkorn U, et al. Impaired cerebral glucose metabolism in prodromal Alzheimer's disease differs by regional intensity normalization. Neurosci Lett. 2013;534:12-17.
Neuropsychiatric Disease and Treatment

\section{Publish your work in this journal}

Neuropsychiatric Disease and Treatment is an international, peerreviewed journal of clinical therapeutics and pharmacology focusing on concise rapid reporting of clinical or pre-clinical studies on a range of neuropsychiatric and neurological disorders. This journal is indexed on PubMed Central, the 'PsycINFO' database and CAS.

\section{Dovepress}

The manuscript management system is completely online and includes a very quick and fair peer-review system, which is all easy to use. Visit http://www.dovepress.com/testimonials.php to read real quotes from published authors. 